\title{
Caracterización de recursos pedagógicos digitales para el desarrollo del pensamiento matemático en un ambiente virtual de aprendizaje
}

\section{Characterization of digital pedagogical resources for the development of mathematical thinking in a virtual learning environment}

\author{
DOI: $10.46932 / s f j d v 2 n 2-195$
}

Received in: March 1st, 2021

Accepted in: May 30th, 2021

\author{
Gilbert Andrés Cruz Rojas \\ Magister en Educación - Universidad del Valle, Cali- Colombia \\ Institución actual: Universidad del Valle \\ Domicilio completo: Cl. 13 \#100-00 Universidad del Valle - Sede Melendez \\ Correo electrónico: gilbert.a.cruz.r@correounivalle.edu.co \\ Edison Alberto Cárdenas Medina \\ Licenciado en Matemáticas y Física. Universidad del Valle, Cali-Colombia. \\ Institución actual: Instituto Tecnológico del Putumayo \\ Domicilio completo: Carrera 2 \#3-69 Barrio Pablo VI San Francisco Putumayo \\ Correo electrónico: edison.cardenas@correounivalle.edu.co \\ Jessica Milena Perdomo Acero \\ Licenciatura en Matemáticas y Física - Universidad del Valle, Cali- Colombia \\ Domicilio completo : Calle 16 \#57 a - 00 Barrio El Limonar - Cali \\ Correo electrónico: jessica.perdomo@ correounivalle.edu.co
}

\section{RESUMEN}

Este documento presenta los avances logrados en el desarrollo del Trabajo de Grado realizado en el área de Educación Matemática del Instituto de Educación y Pedagogía de la Universidad del Valle, Cali Colombia en el año 2019, el cual propone una adaptación de Recursos Pedagógicos en Ambientes de Virtuales de Aprendizaje particularmente los utilizados en el "Diplomado en desarrollo del proceso de pensamiento matemático con mediación de tecnologías", como parte del proceso de oferta e-learning, teniendo en cuenta investigaciones donde se consideran los Recursos Pedagógicos susceptibles a cambios y transformaciones, partiendo de una caracterización y análisis que permita determinar cuáles de estos pueden ser llevados a un Ambiente virtual de Aprendizaje, resolviendo la pregunta ¿Qué tipo de consideraciones didácticas emergen en la adaptación de un conjunto de Recursos Pedagógicos en un Ambiente Virtual de Aprendizaje que posibiliten el desarrollo de procesos del pensamiento matemático? iniciando con la caracterización de los recursos pedagógicos usados en dicho Diplomado.

Palabras clave: Ambiente Virtual de Aprendizaje, Formación Docente, Recursos Pedagógicos.

\begin{abstract}
This document presents the progress made in the development of the Degree Work carried out in the area of Mathematical Education of the Institute of Education and Pedagogy of the Universidad del Valle, Cali - Colombia in 2019, which proposes an adaptation of Pedagogical Resources in Virtual Learning
\end{abstract}


Environments, particularly those used in the "Diploma in the development of the process of mathematical thought with the mediation of technologies". as part of the process of elearning offer, taking into account researches where the Pedagogical Resources are considered susceptible to changes and transformations, starting from a characterization and analysis that allows to determine which of these can be taken to a Virtual Learning Environment, solving the question What type of didactic considerations emerge in the adaptation of a set of Pedagogical Resources in a Virtual Learning Environment that make possible the development of processes of mathematical thought? starting with the characterization of the pedagogical resources used in said Diploma.

Keywords: Virtual Learning Environment, Pedagogical Resources, Teacher Training.

\section{INTRODUCCIÓN}

La educación se ha convertido en un tema importante en Latinoamérica y en especial en Colombia, luego de la Constitución de 1991 y la Ley General de Educación (L. 115/94) se reconocen aspectos para el cuidado y la garantía de la calidad de la educación (Guacaneme E. y Mora L 2011), hay que mencionar además que la educación para los profesores se resalta como importante, como lo han mencionado en varios encuentros (realizados en Valledupar en 1999 y Bogotá en 2003, 2008 y 2011), un coloquio (Bogotá, 2011), al igual que la Asociación Colombiana de Matemática Educativa (Asocolme), en el Encuentro Colombiano de Matemática Educativa (ECME13), así mismo, la Universidad del Valle, ofrece el "Diplomado en desarrollo de procesos centrales de pensamiento con mediación de tecnologías digitales" como parte de la formación continua de los docente.

Sin embargo, la baja cobertura del diplomado hace que muchos docentes interesados en continuar su formación académica no puedan hacerlo, por ende, la oferta de dicho diplomado en la modalidad elearning podría convertirse en una estrategia para continuar el desarrollo de la formación de docentes en Colombia. No obstante, esta modalidad genera algunos interrogantes como: ¿Qué tipo de consideraciones didácticas emergen en la adaptación de un conjunto de Recursos Pedagógicos en un Ambiente Virtual de Aprendizaje que posibiliten el desarrollo de procesos del pensamiento matemático, principalmente en la resolución de problemas?, además ¿Cuál es el conjunto de Recursos Pedagógicos utilizados en el diplomado?, ¿Qué elementos conceptuales están asociados con el uso de Recursos Pedagógicos en un ambiente virtual de aprendizaje? y ¿Cuál es la configuración didáctica de un conjunto de Recursos Pedagógicos del diplomado, en un ambiente virtual de aprendizaje?. En adelante, se busca responder a estos interrogantes mediante el estudio de algunos casos de éxito que han o están ofertando programas académicos o cursos en la modalidad e-learning y de esta forma adaptar dicho conjunto en un ambiente virtual de aprendizaje para posibilitar el desarrollo de proceso del Pensamiento Matemático en la resolución de problemas. 


\section{MARCO DE LA INVESTIGACIÓN}

La Educación en Colombia ha sufrido cambios desde la constitución de 1991, los cuales competen a la formación inicial y continua de los maestros, reconociendo la necesidad de una formación investigativa que favorezca la actitud crítica y la creatividad, además de la integración de Tecnologías de la Investigación y la Comunicación (TIC), de donde resultó que algunos programas de formación inicial desarrollaron actividades complementarias en las asignaturas, y otros incorporaron el uso de la tecnología a sus planes curriculares, mostrando gran interés y preocupación por los elementos que intervienen en la integración de TIC. (Guacaneme, E., Obando, G., Garzón, D., Villa-Ochoa, J. 2013)

Con esta nuevas ideas en educación, se evidencia una necesidad en los docentes de continuar con su formación, para esto, la Universidad del Valle ofrece el "Diplomado en desarrollo de procesos centrales de pensamiento con mediación de tecnologías digitales donde se desarrollan ajustes y estrategias para lograr el objetivo de cada sesión, integrando TIC y elaborando material didáctico que permitan promover procesos centrales del pensamiento matemático de la población escolar, principalmente en resolución de problemas; considerando además que existe un mercado en expansión de los recursos disponibles en Internet que son utilizados por el docente ( Pepin, B., Gueudet, G. \& Trouche, L. 2017) y que en el diplomado son analizados e interpretados, los cuales son adaptados por los docentes debido a que los Recursos Pedagógicos no se consideran terminados y listos para su uso, pues se constituyen como un proceso vivo y de transformación; y se entienden como recorridos distintos, debido a las adaptaciones que se realizan teniendo en cuenta las particularidades del grupo. (Garzon Castro, D y Vega Restrepo, M. 2009).

Respecto a la integración de las herramientas digitales en la Educación Matemática, se considera prometedora y problemática, dado que se reconoce ampliamente el potencial que estas tienen, y se espera que proporcionen nuevos medios de actividades de enseñanza y aprendizaje, aspecto que genera temor pues requiere más dedicación, más tiempo de planeación, evaluación, ajuste y seguimiento, incluso para Chan, M. (2016): la virtualización de la educación y el uso de las TIC, “ es una megatendencia económica y cultural que rebasa el ámbito de la educación escolar" (pp. 3), esto probablemente permita que la modalidad Virtual, se convierta en una gran oportunidad para los docentes, buscando llegar a un equilibrio entre el docente magistral y el docente que se requiere con las nuevas tecnologías, los "Docente Virtuales" denominados así por: Quispe, J. D. E., \& Castro, M. C. H. (2021), para quienes ya están ejerciendo dentro y fuera de la Universidad. 


\subsection{CARACTERIZACIÓN DEL CONTEXTO INSTITUCIONAL.}

En la Universidad del Valle principalmente en el Instituto de Educación y Pedagogía como parte de su mejoramiento a la educación se oferta el "Diplomado en desarrollo de procesos centrales del pensamiento matemático con mediación de tecnologías digitales”, como parte de la política de cualificación docente y la continua formación que se debe brindar en el país, por tal motivo, se ofrece tanto a docentes de la Universidad del Valle como a docentes de otras instituciones, además de profesionales de carreras afines (ingeniería, física, matemática, economía etc), interesados en la educación

El programa se desarrolla de manera semi-presencial en un total de 90 horas, apoyado por el Instituto de Educación y Pedagogía (IEP) y la Dirección de Nuevas Tecnologías y Educación Virtual

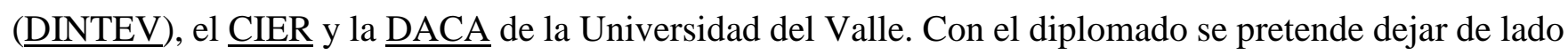
el modelo educativo tradicional, por esta razón se generan innovaciones en la metodología, haciendo uso de actividades en contexto real, hipotético y formal, empleando herramientas digitales y material concreto que permitan el desarrollo de pensamiento matemático, haciendo un trabajo interdisciplinario al integrar varias disciplinas, empleando la tecnología computacional como instrumento de apoyo, y permitiendo de esta manera formar a los docentes discutiendo los cambios que se presentan en la educación matemática.

Hay que mencionar, además que basado en el Plan Estratégico de Desarrollo de la Universidad del Valle existe un compromiso institucional ofreciendo una infraestructura física con salas de cómputo y acceso a Internet, además del soporte tecnológico con la utilización de plataformas virtuales como portal, campus virtual, sistema de información y correo electrónico, debido a que "El curso quedará alojado en las plataformas institucionales de Univalle.” A pesar de esto, se hace necesario profundizar en la utilización de estas plataformas y obtener los beneficios que ofrecen, pues se observa que es poco el aprovechamiento que se está dando con estas herramientas.

\section{METODOLOGÍA}

Para el desarrollo del trabajo se tomó la investigación cualitativa como enfoque metodológico, en donde se adoptó un enfoque descriptivo-interpretativo, el cual se enmarca bajo las dimensiones establecidas por Osses, Sánchez, \& Ibáñez (2006). Se asumieron cuatro momentos en el desarrollo de la metodología: Fundamentación teórica de la sistematización; búsqueda, selección y análisis de los recursos; adaptación y transformación de la situación seleccionada; análisis de resultados.

Para la recolección de información se utilizó una rejilla de análisis, la cual fue retomada del documento La Orquestación Documental, una perspectiva de estudio que permite reflexionar acerca del uso, adaptación y transformación de Recursos Pedagógicos (Henao, A., Arango, H. 2017). La rejilla fue conformada por las unidades de análisis asociadas al recurso y a la situación como tal. De acuerdo a lo 
anterior, es importante mencionar que la rejilla de análisis fue utilizada en dos niveles; en el primer nivel de uso se tomó las sesiones del diplomado y se realizó una caracterización, en donde se logró reconocer los referentes teóricos que sustentaron dicha propuesta, además de identificar algunos recursos empleados por el docente y la estructura de trabajo, realizando preguntas concernientes a la organización didáctica, organización matemática, soportes (materiales), y génesis individuales. En el segundo nivel de uso se pretende caracterizar el proceso de adaptación y transformación de las situaciones seleccionadas. Como elementos más relevantes en este nivel de uso se pretende:

\subsection{DESCRIPCIÓN DE LA PROPUESTA}

El documento que se produjo tiene en cada uno de sus componentes la descripción no solo de lo que se debe hacer sino también de los resultados que se esperan obtener, además se dan a conocer los referentes teóricos que sustentan la propuesta.

\subsection{SITUACIÓN MATEMÁTICA QUE SE PROPONE}

Se caracterizan y escogen los elementos relevantes, vale la pena resaltar que el trabajo varía de una situación a otra en cuanto el trabajo individual o grupal, teniendo en cuenta la adaptabilidad del recurso

\subsection{DIVULGACIÓN DE LOS RESULTADOS DE LA IMPLEMENTACIÓN DE LA PROPUESTA}

Se pone en práctica la situación planteada, el maestro que implementa la situación da a conocer aspectos particulares que pudo haber notado de acuerdo a las características del grupo con el que trabajó y sobre todo de acuerdo a las adecuaciones y transformaciones que él haya realizado al documento inicial.

\subsection{CONSIGNAS DEL TRABAJO A REALIZAR EN TÉRMINOS GENERALES}

Se espera que la actividad seleccionada sea puesta a disposición de los participantes del diplomado, como parte del uso de las plataformas institucionales, permitiendo el acceso a los recursos ahí trabajados y que los estudiantes a partir de las consignas logren dar desarrollo a las situaciones propuestas.

\section{CONCLUSIONES Y RECOMENDACIONES}

De manera general, dado el creciente desarrollo de las TIC en el ámbito educativo, se hace necesario la formación docente, por tanto, se debe asumir un proceso de adaptación a los avances y cambios que genera el mundo constantemente, asumir que la virtualización tarde o temprano llegará a cada sistema educativo actual. Por otra parte, este trabajo contribuye a la implementación adecuada de la 
virtualización de uno o varios recursos pedagógicos, que aportan o dan bases en la construcción de una futura metodología concreta con principios conceptuales y metodológicos que permitan el desarrollo adecuado de dicha virtualización.

La consecución de lo anterior permitiría romper barreras de tiempo y espacio respecto a la educación presencial, generando interés en los docentes que desean continuar su formación profesional y no poseen la facilidad de acceso a las Instalaciones de la Universidad, consecuentemente se aportaría en la mejora de la calidad educativa.

\subsection{CARACTERIZACIÓN Y ANÁLISIS DE RESULTADOS}

El diplomado cuenta con 16 sesiones realizadas semanalmente ( 3 horas por semana) para un total 48 horas presenciales y dos o tres horas de trabajo de campo con una metodología teórico-práctico, realizado mediante una colaboración estratégica entre el Instituto de Educación y Pedagogía (IEP) y la Dirección de Nuevas Tecnologías y Educación Virtual (DINTEV) y el CIER de la Universidad del Valle. Con la ayuda de la rejilla mencionada en el ítem anterior, dicho diplomado se caracterizó en dos partes:

\subsubsection{Primera Parte}

se lograron reconocer tres módulos transversales a dichas sesiones:

\subsubsection{Desarrollo del pensamiento matemático}

Se identificó la estructura de trabajo de la sesión planteada por el profesor, reconociendo los referentes teóricos usados y sus respectivas actividades a desarrollar.

\subsubsection{Uso de tecnología computacional en la enseñanza y el aprendizaje de las matemáticas}

Se observó que el docente usa como herramientas el software: Geogebra y las presentaciones power point.

\subsubsection{Evaluación del aprendizaje en matemáticas}

En su mayoría la evaluación no es explícita durante el desarrollo de las sesiones, sin embargo, se realizan constantes preguntas a los docentes participantes del diplomado, ejercicios para resolver en clase, además de algunas tareas para resolver en casa.

\subsubsection{Segunda Parte}

Se desarrollaron cuatro unidades de análisis con sus respectivas preguntas, de donde se logra 
evidenciar:

En cuanto a la Organización didáctica se puede resaltar que el profesor se apoya en los trabajos de Polya, G., Schoenfeld, A., Mason, J. y Guzmán M., que le permitan el desarrollo de procesos centrales del pensamiento matemático con mediación de tecnologías digitales, particularmente en la resolución de problemas. Además, como parte fundamental del currículo de matemáticas, de igual manera en los lineamientos curriculares teniendo en cuenta los procesos generales de pensamiento, como la modelación, la comparación y ejercitación de procedimientos, el planteamiento y resolución de problemas.

Acerca de la Organización Matemática, el docente privilegia la comunicación, dado que se presentan interrogantes, a lo largo de las actividades de cada sesión, que le permiten realizar un diagnóstico y decidir que estrategias usar para continuar con la clase, muchas de las preguntas exigen un conocimiento básico de algunas definiciones, teoremas y reconocimiento de patrones para la resolución de problemas propuestos en clase, hayan sido resueltos en clase o dejados como tarea.

En relación con los Soportes Materiales, el docente desarrolla su clase con el uso de una presentación Power point y el Software: Geogebra. Cada sesión se está estructurando con mayor detalle, en un libro en construcción por parte del profesor a cargo del Diplomado, material que aún no ha sido publicado y por tanto solo pueden acceder los estudiantes asistentes.

Por último, respecto a las Génesis individuales, el profesor desarrolla las actividades y escoge los problemas que son pertinentes para su clase, donde los problemas se proponen sin que se de ningún tipo de adaptación y/o transformación, es decir se presentan tal y como aparece en el libro en construcción del mismo profesor como autor. Las actividades son desarrolladas, dependiendo de la sesión, de acuerdo a la metodología de los autores mencionados anteriormente y se adaptan al objetivo de la sesión planteada. 


\section{REFERENCIAS}

Benítez, D. (2017). Diplomado en desarrollo de procesos centrales del pensamiento matemático con mediación de tecnologías digitales. Propuesta académica, Universidad del Valle, Instituto de Educación y Pedagogía, Cali.

Chan, M. (2016). La virtualización de la educación superior en América Latina: entre tendencias y paradigmas. RED-Revista de Educación a Distancia, Núm. 48. (Artic. 1.), pp. 1-32. doi: 10.6018/red/48/1

Garzon Castro, D. y. (2009). Los recursos pedagógicos en la enseñanza de la geometría: Estudio de casos. Caracterización de los vínculos entre los recursos pedagógicos y el conocimiento matemático en la enseñanza de las matemáticas en la educación básica. Cali.

Guacaneme, E. y. (2011). La educación del profesor de matemáticas como campo de investigación. Revista PAPELES, Volumen 3(N 6), pp. 18-25.

Guacaneme, E., Obando, G., Garzón, D., \& Villa-Ochoa, J. (2013). Informe sobre la Formación inicial y continua de Profesores de Matemáticas: El caso de Colombia. Cuadernos de Investigación y Formación en Educación Matemática, Especial Noviembre.(8), pp. 11-49.

Henao, A., Arango, H. (2017). La Orquestación Documental, una perspectiva de estudio que permite reflexionar acerca del uso, adaptación y transformación de Recursos Pedagógicos: El caso de "Un grupo de profesores de educación básica primaria de la Institución Educativa Jorge Isaacs”. (Tesis de maestría). Universidad del Valle. Cali, Colombia.

Pepin, B. G. (2017). Refining teacher design capacity: Mathematics teachers' interactions with digital curriculum resources. ZDM Mathematics Education, Volume 49(Issue 5), pp 799- 812. doi: $10.1007 / \mathrm{s} 11858-017-0870-8$

Quispe, J. D. E., \& Castro, M. C. H. (2021). Aplicación de videotutoriales para la mejora de las competencias matemáticas en los estudiantes de pregrado de la Universidad de San Martín de Porres. South Florida Journal of Development, 2(2), 1161-1170.

Trouche, L., \& Drijvers, P. (2014). Webbing and orchestration. Two interrelated views on digital tools in mathematics education. Teaching Mathematics and Its Applications, pp. 1 - 17. doi:10.1093/teamat/hru014 